Nonlinear Processes in Geophysics, 12, 993-1001, 2005

SRef-ID: $1607-7946 / \mathrm{npg} / 2005-12-993$

European Geosciences Union

(C) 2005 Author(s). This work is licensed

under a Creative Commons License.

\title{
Modelling the outer radiation belt as a complex system in a self-organised critical state
}

\author{
N. B. Crosby ${ }^{1, *}$, N. P. Meredith ${ }^{1, * *}$, A. J. Coates ${ }^{1}$, and R. H. A. Iles ${ }^{1}$ \\ ${ }^{1}$ University College London, Mullard Space Science Laboratory, Holmbury St. Mary, Dorking, Surrey, RH5 6NT, UK \\ *now at: Belgian Institute for Space Aeronomy, Ringlaan-3-Avenue Circulaire, 1180 Brussels, Belgium \\ ** now at: British Antarctic Survey, Natural Environment Research Council, Madingley Road, Cambridge, CB3 0ET, UK
}

Received: 28 January 2005 - Revised: 25 July 2005 - Accepted: 19 August 2005 - Published: 11 November 2005

\begin{abstract}
The dynamic behaviour of the outer electron radiation belt makes this area of geo-space a candidate for the concept of self-organized criticality. It is shown here that frequency distributions of measured outer electron radiation belt data are well-represented by power-laws over two decades. Applying the concept of self-organized criticality to interpret the shape of the distributions suggests another approach to complement existing methods in the interpretation of how this complicated environment works. Furthermore sub-grouping the radiation belt count rate data as a function of spatial location or temporal interval (e.g. $L$-shell, magnetic local time, solar cycle, ...) shows systematic trends in the value of the slope of the power-laws. It is shown that the inner part of the outer radiation belt is influenced in a similar manner to the outer part, but in a less profound way. Our results suggest that the entire outer radiation belt appears to be affected as the sum of its individual parts. This type of study also gives the probability of exceeding a given threshold value over a given time; limiting the size of "an event". The average values could then be compared with models used in spacecraft design.
\end{abstract}

\section{Introduction}

The scientific side of space weather is very interdisciplinary, strongly enhancing the links in the chain of physical processes between the Sun (interior, corona and solar wind) and the Earth (magnetosphere, ionosphere, etc.). When a statistical method has been successfully applied in one part of the Sun-Earth system (e.g. the Sun) it may be asked if it could also be successfully applied in another part (e.g. the Earth's outer radiation belt). The concept of self-organized criticality (SOC) is one such method. The original concept of SOC also known as the "avalanche concept", was introduced by Bak et al. $(1987,1988)$, to characterize the behaviour of dissipative

Correspondence to: N. B. Crosby

(norma.crosby@oma.be) systems that contain a large number of elements interacting over a short range. The systems evolve to a critical state in which a minor event starts a chain reaction that can affect any number of elements in the system. It is found that frequency distributions of the output parameters from the chain reaction taken over a period of time can be represented by powerlaws. SOC behaviour has been observed in a large number of phenomena (e.g. sand piles, avalanches, earthquakes, stock markets, solar flares, etc.). It is the area of geo-space which is of interest in this work; how SOC behaviour has been and potentially may be observed in astrophysical phenomena.

With more and more astrophysical data becoming available it is not possible to study each "single event". This is for example the case for large solar flare databases, where contrary to the detailed analysis of individual case study events, statistical studies are performed. The goal of such an approach is to describe the global behaviour of flares by performing frequency distributions of flare parameters. SOC has been a concept used to interpret power-law distributions of large solar flare data bases (e.g. Crosby et al., 1993, 1998; Bromund et al., 1995; Table 1 in Charbonneau et al., 2001). For more information about avalanche models in the context of solar flares see the review paper by Charbonneau et al. (2001). These works have sparked interest in "searching" for SOC in other space phenomena. For example, Gabriel and Feynman (1996) found power-law representations of time-integrated solar energetic particle event fluxes over three to four decades. Lui et al. (2000) investigated whether the dynamic magnetosphere is an avalanching system by performing frequency distributions on parameters of the global auroral distribution. They found that the internal relaxations of the magnetosphere statistically follow powerlaws that have the same index independent of the overall level of activity (quiet vs sub-storm). Watkins et al. (1999) investigated the robustness of collective behaviour in strongly driven magnetospheric avalanche models and found that the crucial power law form of the burst size distribution of large events is surprizingly robust under fast loading. Freeman et al. (2000) showed that the solar wind $v B_{s}$ ( $v=$ solar wind 
Table 1. Characteristics of the electron count rate (CR) frequency distributions for different years and $L$-shell intervals.

\begin{tabular}{clclccc}
\hline Year & $\begin{array}{l}4.1-7.7 \\
\text { interval }\end{array}$ & $\begin{array}{c}4.1-7.7 \\
\text { slope }\end{array}$ & $\begin{array}{l}4.1-5.5 \\
\text { interval }\end{array}$ & $\begin{array}{c}4.1-5.5 \\
\text { slope }\end{array}$ & $\begin{array}{l}5.5-7.7 \\
\text { interval }\end{array}$ & $\begin{array}{c}5.5-7.7 \\
\text { slope }\end{array}$ \\
\hline $1995-1997$ & {$[60,12079]$} & $-1.634 \pm 0.004$ & {$[200,12079]$} & $-1.602 \pm 0.009$ & {$[60,8611]$} & $-1.911 \pm 0.006$ \\
1995 & {$[60,12079]$} & $-1.476 \pm 0.007$ & {$[200,12079]$} & $-1.373 \pm 0.015$ & {$[60,8611]$} & $-1.749 \pm 0.011$ \\
1996 & {$[60,9500]$} & $-1.655 \pm 0.006$ & {$[200,9500]$} & $-1.589 \pm 0.016$ & {$[60,4456]$} & $-1.856 \pm 0.010$ \\
1997 & {$[60,8842]$} & $-1.728 \pm 0.008$ & {$[200,8842]$} & $-1.786 \pm 0.018$ & {$[60,4662]$} & $-2.100 \pm 0.014$ \\
\hline
\end{tabular}

speed, $B_{s}=$ rectified function of the north-south component) and $\epsilon$ burst lifetime distributions are of power law form with an exponential cut-off, consistent with the solar wind being an SOC system. Furthermore, Freeman et al. (2000) showed that the $\epsilon$ burst lifetime distribution is not significantly different to that of the $\mathrm{AU}$ and $|\mathrm{AL}|$ indices, indicating that this scale-free property of the AE indices could arise from the solar wind input and may not be an intrinsic property of the magnetospheric system.

The count rate frequency distribution of more than 5000 hard X-ray structures (less than $1 \mathrm{~s}$ ) detected in 640 solar flares was found to be well-represented by a power-law with a slope of -1.84 (Aschwanden et al., 1995). This is identical with what was found for the global frequency distribution behaviour of solar flare hard X-rays (Crosby et al., 1993). From a scale-invariant model like the avalanche model one would expect that the individual pulses that make up an "avalanche" would also show power-law behaviour and that the slope values would be similar. Even though there are no individually defined "avalanches" in the outer radiation belt, as there are, for example, in the case of the solar corona (e.g. solar flares), one can imagine that the outer radiation belt acts as one individual system "one flare". The analogy is that the pulses that make up the outer radiation belt are identical with the burst structures making up a flare "individual avalanche". Bearing this in mind and the fact that the outer electron radiation belt is indeed dynamical on all spatial and temporal scales, one can speculate if perhaps the outer electron radiation belt also exhibits power-law behavior and thus exibiting itself as a candidate for SOC behaviour. In this paper we present an outer electron radiation belt statistical study based on observations from the Cold Ion Detector (CID) aboard the Space Technolgy Research Vehicle (STRV-1a). For the work the outer electron radiation belt is defined as one entity that changes shape as a function of time.

During the declining phase of the solar cycle, at the time when sunspot minimum is approaching, recurrent high speed solar streams originating from coronal holes occur. These recurrent (due to the Sun's rotation) streams are efficient in driving recurrent magnetic storms and enhancing the radiation belt electrons (Baker et al., 2001). On the other hand, during the ascending phase of the solar cycle, when sunspot maximum is approaching, the occurence of coronal mass ejections (CMEs) increase from about 0.5 to 2 per day. Even though fast CMEs are capable of driving nonrecurrent magnetic storms and accelerating radiation belt electrons, geoeffetive CMEs do not occur as often or as long as the recurrent high speed wind streams do. Geomagnetic disturbances can be monitored by studying changes in the geomagnetic field strength. The $K_{p}$ index (averaged over the whole planet), obtained from a number of magnetometer stations at mid-latitudes, provides information about the overall geomagnetic condition of the ionosphere and its value (on a scale from 0 to 9 ) is highest at times of intense geomagnetic activity.

Past work has indicated that the dynamical aspects of the outer electron radiation belt closely follow the characteristics of the solar wind, especially at times of south-ward interplanetary magnetic field and when solar wind speeds are greater than $450-500 \mathrm{~km} / \mathrm{s}$ (Paulikas et al., 1979; Blake et al., 1997; Li et al., 2001a, b; O'Brien et al., 2001; Iles et al., 2002). Li et al. (2001b) argue that the semiannual variation of $\mathrm{MeV}$ electrons at geostationary orbit is attributed mostly to the semiannual variation of solar wind velocity as seen by Earth. Work performed for the Tsunami consortium looked at the statistical link between spacecraft anomalies and changes in the solar wind and furthermore examined the possible "link" between changes in the solar wind and the "killer electrons" which can have severe commercial consequence (Coates et al., 2002). Here we extend the analysis conducted as part of the Tsunami initiative and take a detailed look at the frequency distributions of the outer radiation belt data. By using the SOC concept additional evidence for the link between the outer radiation belt and the solar wind parameters as well as the $k_{p}$ geomagnetic index is presented here. Furthermore by using the SOC concept we find evidence that the influence of the solar wind velocity is not only observed at geostationary orbit but reaches down to smaller $L$-shells.

The paper is divided into the following: Sect. 2 introduces the CID/STRV-1a experiment, followed by Sect. 3 which presents the statistical studies that are performed on the CID count rate database. In Sect. 4 the results of the statistical study are discussed in context of what they may tell us concerning the dynamics of the outer electron radiation belt and how the results may be used as input for space engineering tools. Section 5 summarizes the conclusions of this work. 


\section{The CID experiment aboard STRV-1a and solar wind data}

The two microsatellites, STRV-1a and 1b, were launched together on 17 June 1994 into geostationary transfer orbit (GTO) with an apogee of $36000 \mathrm{~km}$, perigee of $300 \mathrm{~km}$, orbital period of approximately $11.5 \mathrm{~h}$ and an inclination of $7^{\circ}$. The CID detector aboard STRV-1a was intended to monitor the activity of plasmapheric ions as the spacecraft passed through the Earth's radiation belts (Papatheodorou et al., 1998). The $2 \mathrm{~mm}$ aluminium shielding of the CID detector led to an energy-independent background due to penetrating electrons above approximately $750 \mathrm{keV}$. This background was cross-calibrated with the Radiation Belt Monitor (REM) aboard STRV-1b and the background observed by CID was found to correspond closely with the penetrating high energy electron fluxes observed by REM. By crossing the radiation belts the CID data offers unique information about the dynamics of the electrons that make up the radiation belts in this energy range.

Due to its orbit the STRV-1a spacecraft crossed the radiation belts approximately 4 times per day. CID electron count rate data are available for a $\sim 3.5$ year period from September 1994 until the spacecraft was turned off in March 1998 as a function of $L$-shell ( $L=6371 \mathrm{~km})$, local time (LT) and magnetic field strength. In this study, as a first approximation, LT has been set equal to magnetic local time (MLT). CID detects particles of all pitch angles, but does not resolve them by pitch angle. The time resolution of the count rate data is $6.5 \mathrm{~min}$. For this work we use data from 1995 to 1997.

The electron count rate data is initially binned as a function of $\mathrm{L}$ by averaging in steps of $\Delta L=0.2$ and these data are used in our analysis. Furthermore, we compute the total relativistic electron content $\left(T_{R E C}\right)$ to take into account the non-symmetric aspect of Earth's magnetic field by using the following approximation: $T(L)=C(L) \times L^{2}$, where the factor $L^{2}$ makes an allowance for the volume occupied between dipolar shells $L$ and $L+\Delta L$ (Iles et al., 2002). The $T_{R E C}$ is computed for the following three electron radiation belt intervals: $L=[4.1,7.7], L=[4.1,5.5]$ and $L=[5.5,7.7]$ as a function of day for the three years. The inner $L$-value 4.1 was chosen to best cover the outer electron radiation belt in the most consistent way avoiding "contamination" of the slot region between the outer and inner radiation belt (D. Boscher, private communication). All the count rate profiles have been visually checked, eliminating any that may contaminate the statistical analysis.

Hourly solar wind data (solar wind velocity and interplanetary magnetic field) and the geomagnetic index $K_{p}$ data are downloaded from OMNIweb (http://nssdc.gsfc.nasa.gov/ omniweb/ow.html) for the years 1995-1997. The solar wind data originates from the WIND and IMP-8 spacecraft and there is very high data coverage during these years. OMNIweb obtains the $K_{p}$ index electronically from NOAA's National Geophysical Data Center (ftp://ftp.ngdc.noaa.gov/ STP/GEOMAGNETIC_DATA/INDICES/).

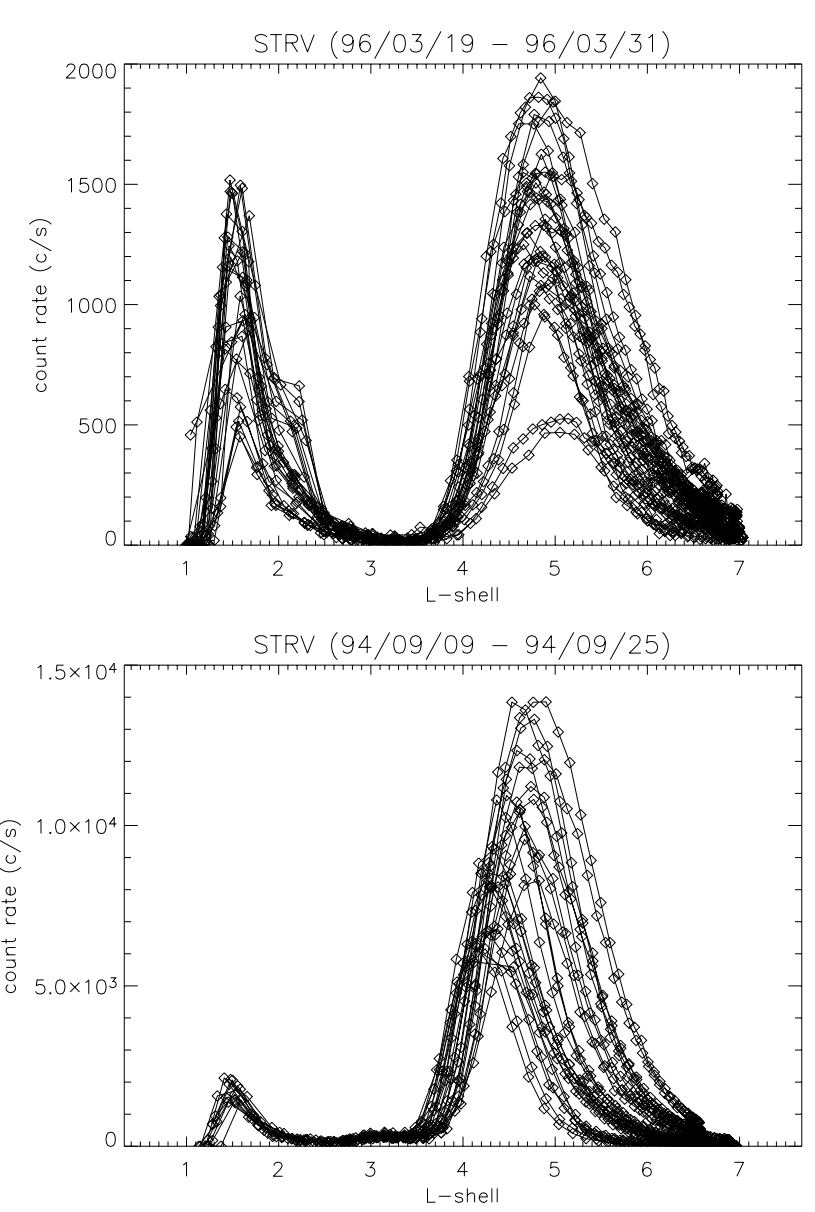

Fig. 1. CID/STRV count rate as a function of $L$-shell for two periods of observation.

Two extended periods of radiation belt electron data $(E>750 \mathrm{keV})$ from the CID STRV-1a observations are shown in Fig. 1. The lower and upper plots show the count rate as a function of $L$-shell for each half-orbit in the period of 9-25 September 1994 and 19-31 March 1996, respectively. The inner and outer electron radiation belts are immediately apparent together with the slot region. The upper plot shows that the maximum count rate of the outer belt varies between 500 and $2000 \mathrm{c} / \mathrm{s}$ (a factor of four). On the other hand during another period of observation (lower plot) the maximum count rate varies between 5000 and $\sim 14000 \mathrm{c} / \mathrm{s}$. Although the factor is less than three (factor four in upper plot), the total variability between the two plots (500 and $\sim 14000 \mathrm{c} / \mathrm{s}$ gives a factor of 28 . The plots also show how the boundaries of the radiation belt changes over time as it expands and contracts. Indeed, these observations emphasize the dynamical aspect of the radiation belts on all spatial and temporal scales.

\section{Statistical analysis of data}

The orbit of the STRV-1a spacecraft as a function of $L$-shell and MLT is plotted for the three years: Fig. 2 (1995), Fig. 3 


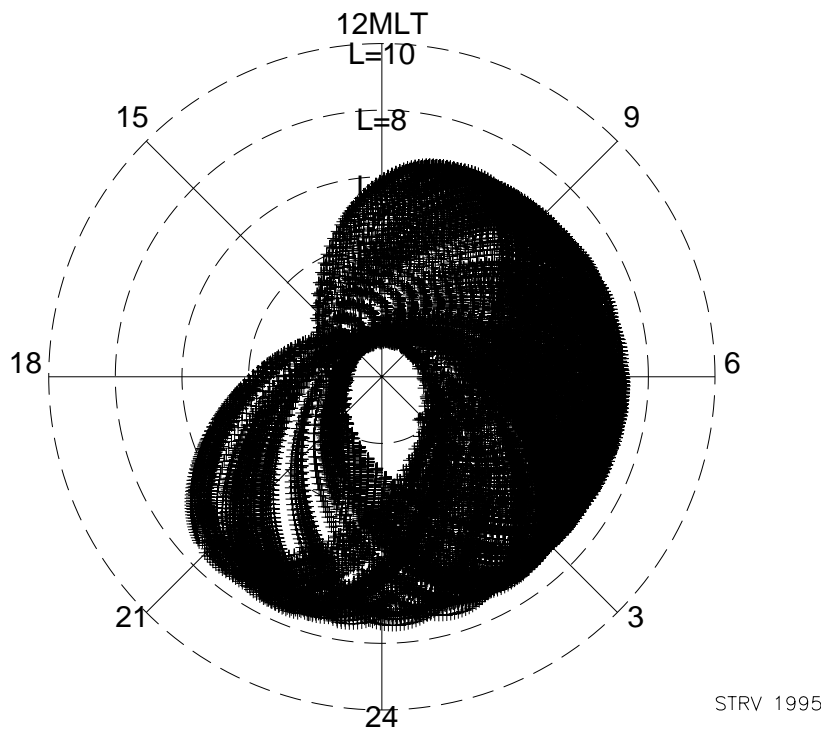

Fig. 2. 1995 STRV orbit (function of $L$-shell and MLT).

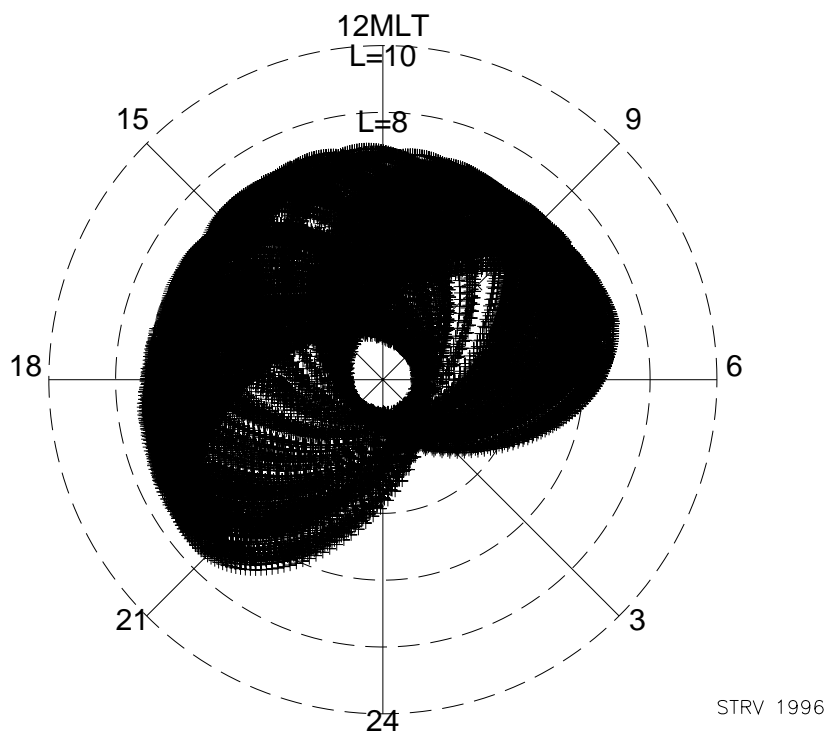

Fig. 3. 1996 STRV orbit (function of $L$-shell and MLT).

(1996) and Fig. 4 (1997). A "spatial span” of 13 MLT hours (00:00-10:00 MLT and 21:00-24:00 MLT) is covered by the spacecraft's orbit for 1995. For 1996 the spacecraft's coverage is 07:00-22:00 MLT and for 1997 (00:00-07:00 MLT and 19:00-24:00 MLT). To take into account any possible effect that the MLT location may have on the radiation belt data, each year is considered separately as well as together. In this way any influence that the solar cycle variation may have on the radiation belt data is also included.

For our statistical analysis we present frequency distributions (log-log plots) of the following parameters: electron count rate, total relativistic electron content, solar wind velocity and geomagnetic $K_{p}$ index. What is illustrated in the figures is the number of events that fall into a given interval

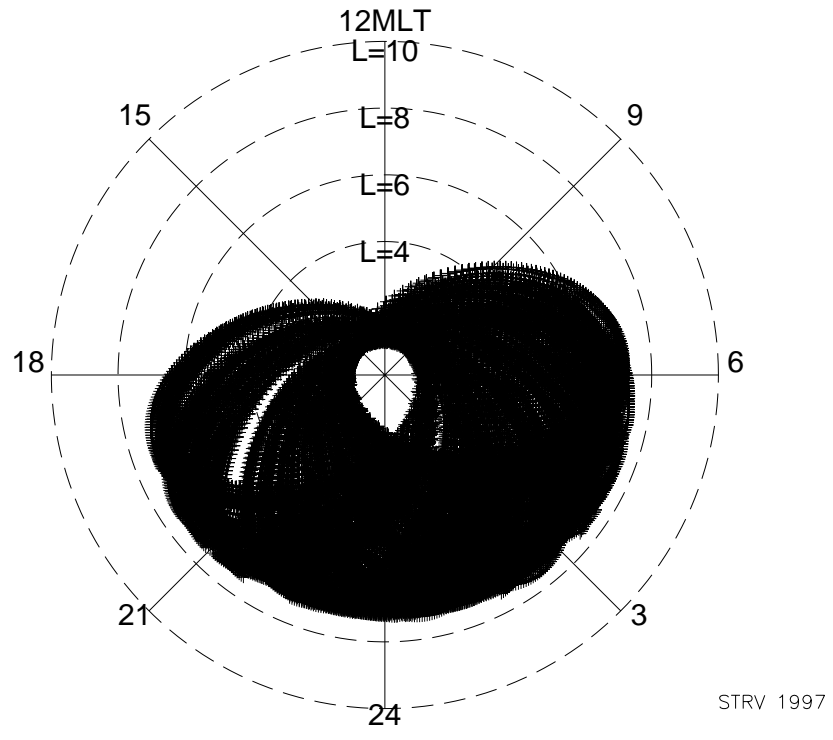

Fig. 4. 1997 STRV orbit (function of $L$-shell and MLT).

divided by the width of the interval. It should be emphasized that the histogram profile illustrating the frequency distribution on the figures is to aid visualization and that when we compute the slope of the power-law representation a maximum likelihood method is used that includes all the individual data-points.

Figure 5 illustrates the frequency distributions of the CID/STRV-1a count rate (CR) data for the three years (1995, 1996 and 1997) and three $L$-shell intervals: Left column: $L=[4.1,7.7]$, Middle column: $L=[4.1,5.5]$ and Right column: $L=[5.5,7.7]$. The figures show that the higher end of the frequency distribution is wellrepresented by a power-law between two and two and a half decades. The slopes found for the three $L$-shell intervals in 1995 are: upper left panel $(\alpha=-1.476 \pm 0.007)$, upper middle panel $(\alpha=-1.373 \pm 0.015)$ and upper right panel $(\alpha=-1.749 \pm 0.011)$. The power-law behavior starts significantly higher $(\mathrm{CR}=200 \mathrm{c} / \mathrm{s})$ for the interval $L=[4.1,5.5]$ compared to the interval $L=[5.5,7.7]$ and the total interval $L=[4.1,7.7]$, where it starts at $\mathrm{CR}=60 \mathrm{c} / \mathrm{s}$; similar behaviour is observed for the other two years (1996 and 1997).

The slopes found for the two $L$-shell intervals $L=[4.1,7.7]$ and $L=[4.1,5.5]$ are very similar for each of the three years, whereas the slope for the outer $L$-shell interval $L=[5.5,7.7]$ is systematically steeper. Results, including the range of the fits, from the analysis for the three years are listed in Table 1, including the results for all three years analyzed together. The slope for all three $L$-shell intervals is at minimum in 1995 and increases systematically as a function of year.

Analyzing all three years together we divide the data for $L=[4.1,7.7]$ into four subgroups as a function of MLT $=[00: 00-06: 00], \quad[06: 00-12: 00], \quad[12: 00-18: 00]$ and [18:00-24:00]. Frequency distributions of the four subgroups are shown on Fig. 6 and all four distributions 
Table 2. Characteristics of the electron count rate (CR) frequency distributions for different MLT intervals for $1995-1997$.

\begin{tabular}{clclccc}
\hline MLT & $\begin{array}{l}4.1-7.7 \\
\text { interval }\end{array}$ & $\begin{array}{c}4.1-7.7 \\
\text { slope }\end{array}$ & $\begin{array}{l}4.4-5.5 \\
\text { interval }\end{array}$ & $\begin{array}{c}4.1-5.5 \\
\text { slope }\end{array}$ & $\begin{array}{l}5.5-7.7 \\
\text { interval }\end{array}$ & $\begin{array}{c}5.5-7.7 \\
\text { slope }\end{array}$ \\
\hline $0-6$ & {$[60,12079]$} & $-1.674 \pm 0.007$ & {$[150,12079]$} & $-1.517 \pm 0.015$ & {$[60,7609]$} & $-1.959 \pm 0.015$ \\
$6-12$ & {$[60,11837]$} & $-1.522 \pm 0.007$ & {$[150,11837]$} & $-1.319 \pm 0.014$ & {$[60,5345]$} & $-1.705 \pm 0.010$ \\
$12-18$ & {$[60,9500]$} & $-1.540 \pm 0.010$ & {$[150,9500]$} & $-1.476 \pm 0.019$ & {$[60,4456]$} & $-1.824 \pm 0.018$ \\
$18-24$ & {$[60,8611]$} & $-1.803 \pm 0.010$ & {$[150,7815]$} & $-1.819 \pm 0.019$ & {$[60,8611]$} & $-2.234 \pm 0.017$ \\
\hline
\end{tabular}

Table 3. Characteristics of the total relativistic electron content $\left(T_{R E C}\right)$ frequency distributions for different years and $L$-shell intervals.

\begin{tabular}{clclclc}
\hline Year & $\begin{array}{l}4.1-7.7 \\
\text { interval }\end{array}$ & $\begin{array}{c}4.1-7.7 \\
\text { slope }\end{array}$ & $\begin{array}{l}4.1-5.5 \\
\text { interval }\end{array}$ & $\begin{array}{c}4.1-5.5 \\
\text { slope }\end{array}$ & $\begin{array}{l}5.5-7.7 \\
\text { interval }\end{array}$ & $\begin{array}{c}5.5-7.7 \\
\text { slope }\end{array}$ \\
\hline 1995 & {$[8000,359717]$} & $-1.195 \pm 0.060$ & {$[8000,274153]$} & $-1.254 \pm 0.069$ & {$[8000,111914]$} & $-1.548 \pm 0.122$ \\
1996 & {$[8000,270400]$} & $-1.535 \pm 0.082$ & {$[8000,199090]$} & $-1.430 \pm 0.104$ & {$[8000,71309]$} & $-2.074 \pm 0.193$ \\
1997 & {$[8000,216389]$} & $-1.739 \pm 0.092$ & {$[8000,160989]$} & $-1.950 \pm 0.119$ & {$[8000,109188]$} & $-2.675 \pm 0.234$ \\
\hline
\end{tabular}

Table 4. Characteristics for the $K_{p}$ geomagnetic index frequency distributions for different years (1995: full line, 1996: dotted line, 1997: dashed line).

\begin{tabular}{cc}
\hline $\begin{array}{c}K_{p} \text { index } \\
\text { YEAR }\end{array}$ & slope \\
\hline 1995 & $-1.424 \pm 0.019$ \\
1996 & $-1.716 \pm 0.021$ \\
1997 & $-1.825 \pm 0.023$ \\
\hline
\end{tabular}

are well-represented by a power-law over two decades. The slopes found for the four distributions are (from top to botttom): MLT $=[00: 00-06: 00] \quad(\alpha=-1.674 \pm 0.007)$, MLT $=[06: 00-12: 00] \quad(\alpha=-1.522 \pm 0.007) \quad$ MLT $=[12: 00-$ 18:00] $\quad(\alpha=-1.540 \pm 0.010)$ and MLT $=[18: 00-24: 00]$ $(\alpha=-1.803 \pm 0.010)$. A similar analysis is performed on the other two $L$-shell intervals: $L=[4.1,5.5]$ and $L=[5.5,7.7]$. The computed slope values are listed in Table 2 and for all three $L$-shell intervals the MLT interval [18:00-24:00] slope is significantly steeper than it is for the other three MLT intervals.

Figure 7 illustrates the frequency distributions of the CID/STRV-1a $T_{R E C}$ data for the three years (1995, 1996 and 1997) and the three $L$-shell intervals: Left Panels: $L=[4.1,7.7]$, Middle Panels: $L=[4.1,5.5]$ and Right Panels: $L=[5.5,7.7]$. The figures show that the higher end of the frequency distribution is well-represented by a power-law over one decade and the computed slopes of the power-law representation are listed in Table 3. As was seen in the CR data
Table 5. Characteristics for the solar wind velocity $\left(V_{s w}\right)$ frequency distributions for different years and interplanetary magnetic field intervals.

\begin{tabular}{cccc}
\hline YEAR & $\begin{array}{c}V_{s w} \\
(-B z+B z)\end{array}$ & $\begin{array}{c}V_{s w} \\
(-B z)\end{array}$ & $\begin{array}{c}V_{s w} \\
(+B z)\end{array}$ \\
\hline 1995 & $-5.54 \pm 0.15$ & $-5.66 \pm 0.19$ & $-5.40 \pm 0.26$ \\
1996 & $-10.45 \pm 0.27$ & $-10.59 \pm 0.35$ & $-10.27 \pm 0.43$ \\
1997 & $-13.76 \pm 0.59$ & $-13.44 \pm 0.74$ & $-14.32 \pm 0.99$ \\
\hline
\end{tabular}

(Fig. 5) the slope for all three $L$-shell intervals is at minimum in 1995 and increases systematically as a function of year. The power-law behavior starts around $\left(T_{R E C}=8000 \mathrm{c} / \mathrm{s}\right)$ for all three intervals as well as for all three years.

Figure 8 shows the frequency distribution of the geomagnetic $K_{p}$ index for the three years (1995: full line, 1996: dotted line, 1997: dashed line). The power-law fits for the three years are listed in Table 4. There is a significant steepening of the slope value from $1995(\alpha=-1.424 \pm 0.019)$ to $1997(\alpha=-1.825 \pm 0.023)$.

Frequency distributions of the solar wind velocity ( $V_{s w}$ for the three years $(1995,1996$ and 1997) are plotted in Fig. 9 for all data (left panels) and for two sub-groups of values: 1) corresponding to southward interplanetary magnetic field (middle panels) and 2) corresponding to northward interplanetary magnetic field (right panels). For each plot the powerlaw fit of the solar wind velocity distribution for the interval $(500 \mathrm{~km} / \mathrm{s}, 900 \mathrm{~km} / \mathrm{s})$ is computed again using the maximum likelihood method. Results for the three years are listed in Table 5. Slopes for all three interplanetary magnetic field in- 

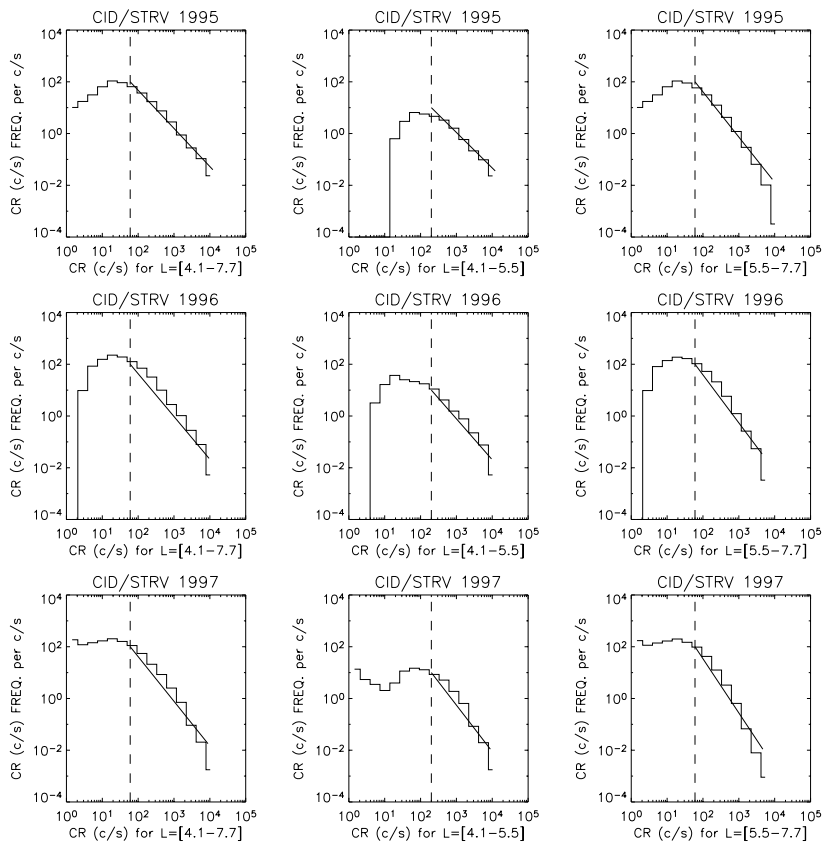

Fig. 5. Frequency distributions of the outer radiation belt electron count rate data (CID/STRV-1a) for 1995, 1996 and 1997. The $L$-shell interval is $[4.1,7.7]$ for the left column, [4.1, 5.5] for the middle column and $[5.5,7.7]$ for the right column.
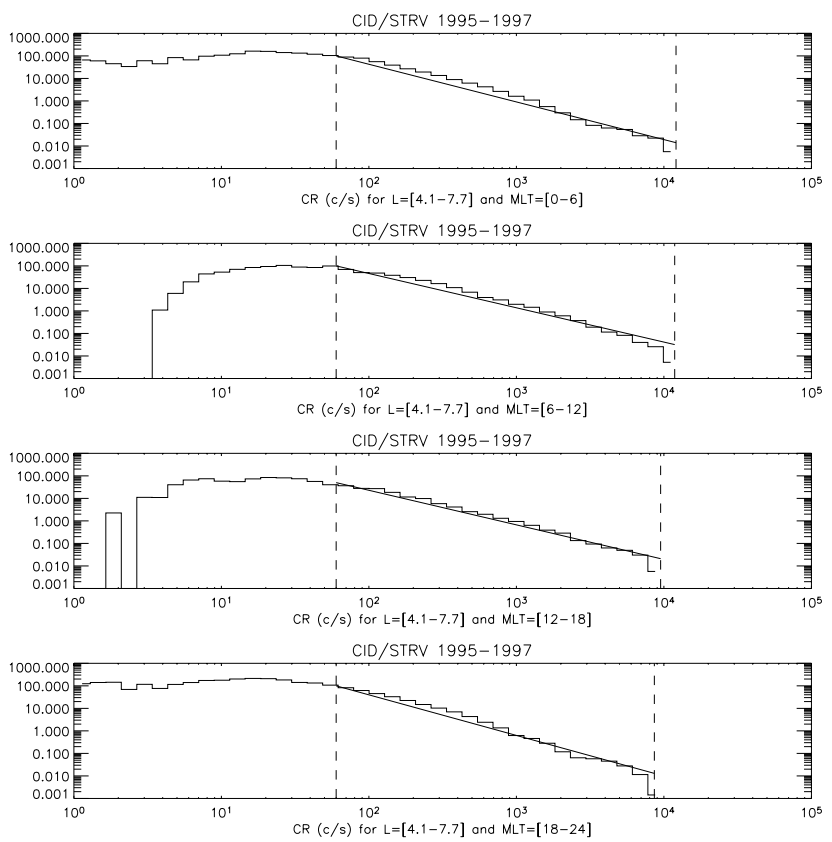

Fig. 6. Frequency distributions of all the outer radiation belt electron count rate data (CID/STRV-1a) for the $L$-shell interval [4.1, 7.7] as a function of MLT: [0-6], [6-12], [12-18], [18-24].

tervals (all data, southward interplanetary magnetic field and northward interplanetary magnetic field) are smallest in 1995 and increase systematically as a function of year. The shapes of the three sub-groups are similar, though the southward in-
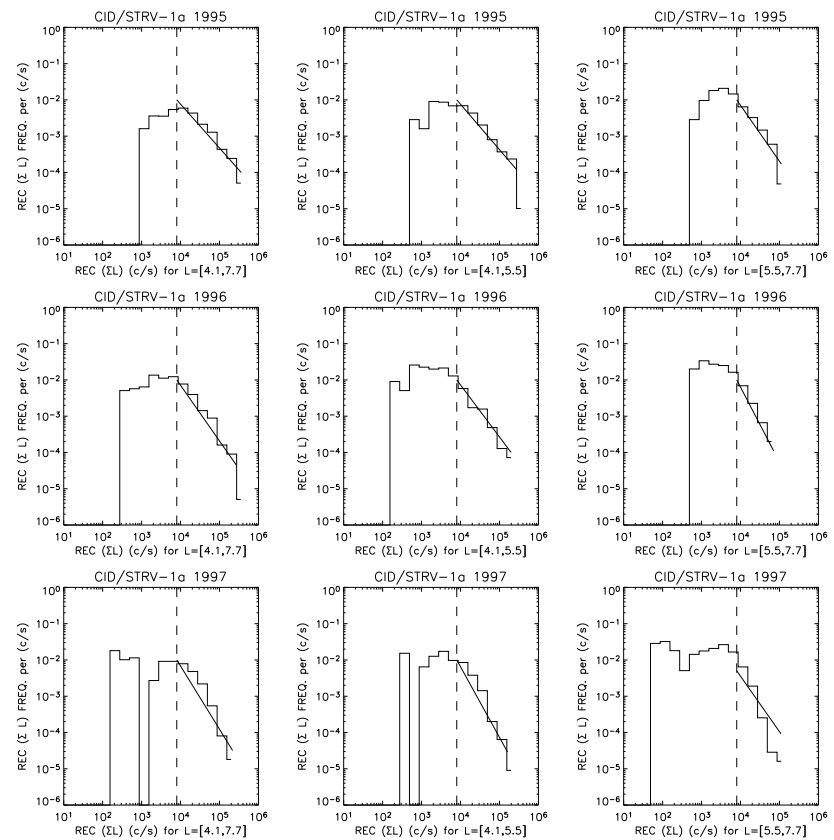

Fig. 7. Frequency distributions of the outer radiation belt total relativistic electron content data (CID/STRV-1a). The $L$-shell interval is $[4.1,7.7]$ for the left-hand panels, $[4.1,5.5]$ for the middle panels and $[5.5,7.7]$ for the right-hand panels.

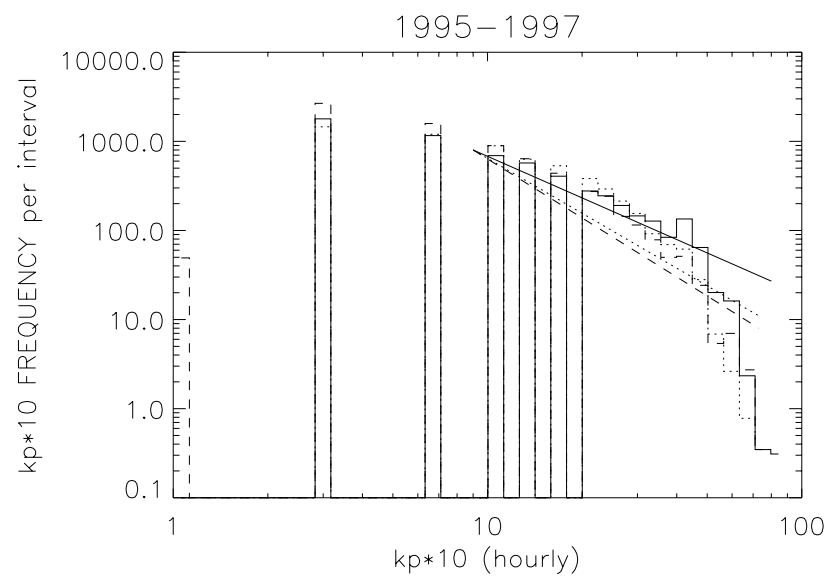

Fig. 8. $K_{p}$ geomagnetic index frequency distributions for different years (1995: full line, 1996: dotted line, 1997: dashed line).

terplanetary magnetic field distribution is overall larger than its corresponding northward counterpart.

\section{Discussion}

In 1995 when recurrent high speed solar wind streams are present the slope of both the CR and $T_{R E C}$ frequency distribution is at its lowest value indicating that the upper part of the frequency distribution dominates the overall distribution. This is supported by the similar trend in the geomagntic index $K_{p}$ frequency distribution indicating more large- 
scale geomagetic activity. The effect dissapears as the slope systematically increases during the next two years. The fact that the slope is different in 1995 and 1997, where the observations originate from similar "spatial span" MLT intervals, suggests that the increase in slope is solar cycle dependent rather than MLT dependent.

For the CR frequency distribution the slopes for the two $L$-shell intervals $(L=[4.1,7.7]$ and $L=[4.1,5.5])$ are similar in most of the cases, whereas, the $L=[5.5,7.7]$ slope value is systematically steeper. The slope value for the $T_{R E C}$ frequency distributions for the two $L$-shell intervals $(L=[4.1,7.7]$ and $L=[4.1,5.5])$ are also similar in most of the cases and, again, the $L=[5.5,7.7]$ slope value is systematically steeper. This is due to the fact that we see a greater range of variation in the inner region and, in particular, a greater frequency of larger count rates which leads to a shallower slope in the inner region.

The above indicates that the entire outer radiation belt appears to be affected as the sum of its individual parts and suggests that the magnitude that the external disturbances influence are a function of $L$-shell.

When sub-grouping the CR data as a function of MLT it is found that the slopes computed for the two morning sector sub-groups $[6,12]$ and $[12,18]$ are flatter than the two night sectors $[0,6]$ and $[18,24]$. The slope of the sub-group MLT $=[18,24]$ (Table 2) is very similar to the one found for the 1997 CR (Table 1) suggesting that the differences in the MLT sub-group slopes may be an effect of the solar cycle. As the electrons have a drift period of around $15 \mathrm{~min}$, one would imagine that there does not exist significant differences in the sub-group MLT behaviour.

For all three years the power-law behaviour for the CR frequency distribution is most pronounced (observed over a larger range) for the outer region of the outer radiation belt interval $L=[5.5,7.7]$ in contrast to the inner region of the outer radiation belt interval $L=[4.1,5.5]$. This is evidence for more dynamic control in the outermost region of the outer radiation belt. Geoeffective strong/moderate storms may effect the whole of the outer radiation belt, peaking in the range $4<L<5$, wheras weaker storms and periods of prolonged substorm activity in the absence of a magnetic storm signature tend to be only effective further out, peaking typically in the range $5<L<6$, (Meredith et al., 2002). Thus geoeffective storms will contribute to enhancements in the outer region $(5.1<L<7.7)$ of the outer radiation belt whereas only the strong/moderate geoeffective storms will effect the inner region of the outer radiation belt $(4.1<L<5.5)$. This may contribute to the result that there appears to be a more dynamic control in the outer region. This effect is less easy to establish in the the $T_{R E C}$ frequency distributions.

The power-law slope behaviour found for both the CR and the $T_{R E C}$ frequency distributions is similar to what has been found for other astro-physical phenomena (e.g. solar flares, substorms, etc.). Also the slope value is similar to (lies in the range of) what has been found in these statistical studies and others in the literature. Results suggest that the modelling of the radiation belt as a complex system in a self-
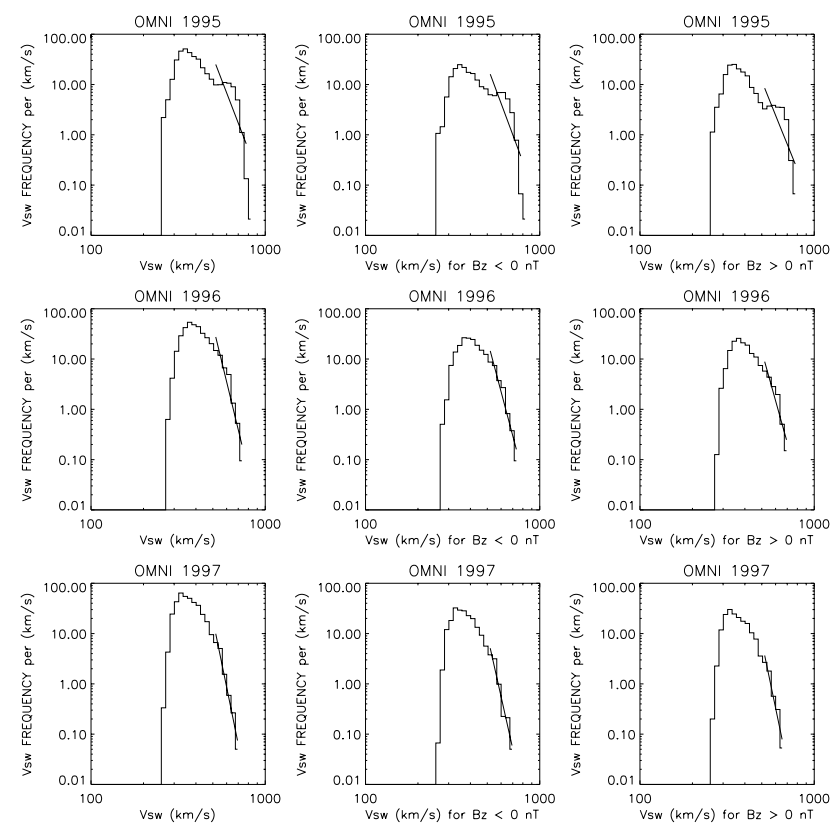

Fig. 9. Solar wind velocity $\left(V_{s w}\right)$ frequency distributions for 1995 , 1996 and 1997 (left panels: all data, middel panels: negative interplanetary field association, right panels: positive interplanetary field association).

organized critical state provides a good context to understand the frequency distributions of the electron parameters. The turn-over in the distributions for small count rates could be attributed to the reduced efffieciency of finding "events" in the error bar range of the "background" and/or simply the fact that the "background" is included in the chosen $L$-range. Similar interpretations are for example used for solar flare small count rate distribution turn-overs (e.g. Crosby et al., 1993).

For this work the solar wind velocity frequency distribution was also fitted by a power-law and the results are shown in Fig. 9. The fit is not so good for the 1995 solar wind data and you can clearly see two populations in the upper panels of the figure. The population with the higher velocities are related to the high speed solar wind streams. A systematic increase in the slope value as a function of year (minimum in 1995) is observed and emphasizes that high-speed solar winds dominate the distribution in 1995. Looking at the whole solar wind velocity database (left panel) or only data with either negative (middle column) or positive (right column) interplanetary magetic field does not change the solar wind velocity distribution within error bars. This tells us that statistics of the overall solar wind velocity can be used when investigating the possible link between the solar wind and the outer radiation belt. The fact that the power-law slope value systematically increases as a function of year for both the solar wind velocity (Vsw) and the electron count rate (CR) as well as for the total relativistic electron content $\left(T_{R E C}\right)$ suggests a connection between the solar wind and the radiation belts. This also suggests that the solar wind is an important driver of the overall statistics of the outer radiation belt. 
Burlaga and Lazuras (2000) found that the lognormal distribution is a good model for the solar wind velocity data measured in 1995 to 1998 (less satisfactory for the data obtained during 1995). We do not suggest that our power-law distribution is a better representation of the statistical properties observed in the solar wind velocity data or for the geomagnetic index $K_{p}$. Instead we want to look for trends in the slopes and emphasize, using these results, that any realistic fit would show tendencies in the statistical properties of the data during the solar cycle. Hence the signature (e.g. power-law slope) of the statistical properties of the data as a function of year would be observed in any type of fit to the data.

The importance in understanding the dynamical behaviour of the outer electron radiation belt is important for the design of spacecraft crossing this region (once or numerous times) and of spacecraft situated in geostationary orbit. Traditional simulation of the radiation belts has relied on empirical models and apart from separate versions for solar minimum and solar maximum does not include the dynamic nature throughout the solar cycle on all time-scales. As long as there is enough statistics the SOC method provides information on the statistical properties of the variability that is present in the system being observed (e.g. electron count rate (CR), total relativistic electron content $\left.\left(T_{R E C}\right), \ldots\right)$ on any chosen time-scale e.g. months, years, decades, ...). Furthermore this type of study also gives the probability of exceeding a given threshold value over a given time; limiting the size of "an event". The average values can then be compared with models used in spacecraft design.

Xapsos et al. (2000) developed a model predicting cumulative solar proton event fluence distributions using the Maximum Entropy approach and results correpond well with the measured solar proton distributions. Another study using extreme value analysis analysis was applied to the magnetic index $A_{p}$, solar proton daily averaged flux values, as well as to the exceedances of $>2 \mathrm{MeV}$ electron flux values over a threshold value at geosynchronous orbit (Koons, 2001). Results show that the extreme values observed to date are not unusual in that they are well fit by extreme value models. They also show that larger values than observed to date can be expected for each of the parameters during any 100-year period. Similar types of analysis could be performed on data originating from the "total" outer electron radiation belt.

\section{Conclusions and prospectives}

For the first time the SOC approach has been applied to outer electron radiation belt data. The main results of the work are the following:

- Modelling of the outer radiation belt as a complex system in a self-organized critical state provides a good context to understand the frequency distributions of the electron parameters.
- There is evidence for more dynamic control by the solar wind in the outer region of the outer radiation belt.

- The entire outer radiation belt appears to be affected as the sum of its individual parts.

- A link between the solar wind characteristics and the outer radiation belt dynamics is suggested by this technique in agreement with earlier studies which used different techniques.

From a scientific point-of-view these results support the fact that the outer electron radiation belt can be analysed as one entity (there is coherence in the system). This way of describing the system allows one to see how disturbances (using the $K_{p}$ geomagnetic index as an indicator) from the outside can take the system (outer electron radiation belt) to the edge of chaos and then cascade it into a new order "organization".

On-going work using radiation belt electron count rate data from the HEO-3 spacecraft, also finds evidence for power-law behaviour (Crosby et al., 2005 ${ }^{1}$ ). This supports the above results presented in this paper.

Statistical results such as these also offer some information which are useful for engineering tools: the probability of extreme events (limit to the size of an "event" over a given time period). Under all circumstances any successful empirical or theoretical radiation belt model should be able to explain (or at least to reproduce) the results of statistical studies performed on outer electron radiation belt data.

Acknowledgements. This work was supported by PPARC, and is an extension of the work performed for the Tsunami consortium. BIRA's contribution to this work was done in the context of the "Living with a Star" program and PRODEX contract no. $14731 / 00 / \mathrm{NL} / \mathrm{SFe}(\mathrm{IC})$. This paper has used data obtained from the NSSDC OMNIWeb database.

Edited by: P.-L. Sulem

Reviewed by: S. McIntosh and another referee

\section{References}

Aschwanden, M. J., Schwartz, R. A., and Alt, D. M.: Electron timeof-flight differences in solar flares, Astrophys. J., 447, 923-935, 1995.

Bak, P., Tang, C., and Wiesenfeld, K.: Self-organized criticality An explanation of 1/f noise, Phys. Rev. Lett., 59, 381-384, 1987.

Bak, P., Tang, C., and Wiesenfeld, K.: Self-organized criticality, Phys. Rev. A, 38/1, 364-374, 1988.

Baker, D. N., Kanekal, S. G., Blake, J. B., and Pulkkinen, T. I.: The global efficiency of relativistic electron production in the Earth's magnetosphere J. Geophys. Res., 106, A9, 19 169-19 178, 2001.

Blake, J. B., Baker, D. N., Turner, N., Ogilvie, K. W., and Lepping, R. P.: Correlation of changes in the outer-zone relativisticelectron population with upstream solar wind and magnetic field measurements, Geophys. Res. Lett., 24, 8, 927-929, 1997.

\footnotetext{
${ }^{1}$ Crosby, N. B, Fennell, J. F, and Heynderickx, D.: in preparation, 2005.
} 
Bromund, K. R., McTiernan, J. M., and Kane, S. R.: Statistical studies of ISEE 3/ICE observations of impulsive hard X-ray solar flares, Astrophys. J., 455, 733-745, 1995.

Burlaga, L. F. and Lazarus, A. J.: Lognormal distributions and spectra of solar wind plasma fluctuations: Wind 1995-1998, J. Geophys. Res., 105, A2, 2357-2364, 2000.

Charbonneau, P., McIntosh S. W., Liu, H.-L., and Bogdan T. J.: Avalanche models for solar flares (Invited Review), Solar Physics, 203, 2, 321-353, 2001.

Coates, A. J., Crosby, N. B., Linder, D. R., Kataria, D. O.: Space weather studies for the satellite insurance industry, Proceedings of Space Weather Workshop: Looking towards a European Space Weather Programme, http://www.esa-spaceweather.net/spweather/workshops/ SPW_W3/PROCEEDINGS_W3/index.html, 2001.

Crosby, N. B., Aschwanden, M. J., and Dennis, B. R.: Frequency distributions and correlations of solar X-ray flare parameters, Solar Physics, 143, 2, 275-299, 1993.

Crosby, N. B, Vilmer, N., Lund, N., and Sunyaev, R.: Deka-keV $\mathrm{X}$-ray observations of solar bursts with WATCH/GRANAT: frequency distributions of burst parameters, Astron. \& Astrophys., 334, 299-313, 1998.

Freeman, M. P., Watkins, N. W, and Riley, D. J.: Evidence for a solar wind origin of the power law burst lifetime distribution of the AE indices, Geophys. Res. Lett., 27, 8, 1087-1090, 2000.

Gabriel, S. B., and Feynman, J.: Power-law distribution for solar energetic proton events, Solar Phys., 165, 337-346, 1995.

Iles, R.H. A., Fazakerley, A. N, Johnstone, A. D., Meredith, N. P., and Bühler, P.: The relativistic electron response in the outer radiation belt during magnetic storms, Ann. Geophys., 20, 7, 957965, SRef-ID: 1432-0576/ag/2002-20-957, 2002.

Koons, H. C.: Statistical analysis of extreme values in space science, J. Geophys. Res., 106, A6, 10915-10 921, 2001.

Li, X., Temerin, M., Baker, D, N., Reeves, G. D., and Larson, D.: Quantitative prediction of radiation belt electrons at geostationary orbit based on solar wind measurements, Geophys. Res. Lett., 28, 9, 1887-1890, 2001.
Li, X., Baker, D. N, Kanekal, S. G, Looper, M., and Temerin, M.: Long term measurements of radiation belts by SAMPEX and their variations, Geophys. Res. Lett., 28, 20, 3827-3830, 2001.

Lui, A.T.Y, Chapman, S. C., Liou, K., Newell, P. T., Meng, C. I., Brittnacher, M., and Parks, G.: Is the dynamic magnetosphere an avalanching system?, Geophys. Res. Lett., 27, 7, 911-914, 2000.

Meredith, N. P., Cain, M., Horne, R. B, Thorne, R. M., Summers, D., and Anderson, R. R.: Evidence for chorus-driven electron acceleration to relativistic energies from a survey of geomagnetically disturbed periods, J. Geophys. Res. Space Physics, 108, A6, 1248, doi:10.1029/2002JA009764, 2003.

O'Brien, T. P., McPherron, R. L, Sornette, D., Reeves, G. D., Friedel, R., and Singer, H. J.: Which magnetic storms produce relativistic electrons at geosynchronous orbit?, J. Geophys. Res., 106, 8, 15 533-15 544, 2001.

Papatheodorou, G., Woodliffe, R. D., Johnstone, A. D., Rodgers, D. J., Walton, D. M. and Sims, A. J.: A miniature retarding potential plasma analyser with a differential energy response, Measurement Techniques in Space Plasmas, in: AGU monograph, edited by: Pfaff, R. F., Borovsky, J. E., Yung, D. T., 102, 287293, 1998.

Paulikas, G. A. and Blake, J. B.: Effects of the solar wind on magnetospheric dynamics: energetic electrons at the synchronous orbit, in: Quantitative Modeling of Magnetospheric Processes, edited by: Olson, W. P., 21, 180-202, 1979.

Watkins, N. W., Chapman, S. C, Dendy, R. O., and Rowlands, G.: Robustness of collective behaviour in strongly driven avalanche models: magnetospheric implications, Geophys. Res. Lett., 26, 16, 2617-2620, 1999.

Xapos, M. A., Summers, G. P, Barth, J. L., Stassinopoulos E. G., and Burke, E. A.: Probability model for cumulative solar proton event fluences, IEEE Trans. Nucl. Sci., 47, 3, 486-490, 2000. 